\title{
Épistémologies pratiques du professeur et des élèves. Une étude de l'enseignement par débats d'idées au cours des jeux sportifs collectifs à l'école primaire
}

\author{
Antoine Thépaut \\ Antoine Thépaut. \\ Résumé. L'objet de l'étude présentée ici explore quelques-unes des dimensions de l'épistémologie pratique \\ du professeur et des élèves pour l'analyse du processus d'enseignement-apprentissage en EPS à l'école \\ élémentaire, ici en jeux sportifs collectifs. Cette étude met à jour l'effet de levier qu'entraîne l'introduction d'un \\ geste didactique de métier familier chez les PE : le débat d'idées pour initier des pratiques d'enseignement \\ novatrices. Elle souligne la pertinence d'un approfondissement de l'épistémologie pratique pour identifier les \\ contraintes qui pèsent sur la transmission des contenus.
}

Mots-clés. Épistémologie pratique, Éducation physique et sportive, École primaire, Sports collectifs

\section{Introduction}

L'objet de notre communication vise à explorer quelques-unes des dimensions de "l'épistémologie pratique du professeur et des élèves » émergeant lors de l'enseignement des jeux sportifs à l'école élémentaire. Le concept d'épistémologie pratique retravaillé par Amade-Escot fait actuellement l'objet de discussions et de débats visant à en déployer la portée explicative (Amade-Escot, 2014, 2019; Cicurel, 2019). Nous cherchons ici à cerner la portée générale de ce concept pour une compréhension du fonctionnement du système didactique lors de l'enseignement des jeux sportifs collectifs et de l'EPS à l'école primaire de façon plus générale. Cette approche repose sur l'étude en intelligibilité des savoirs des enseignants. Classiquement il est considéré que les professeurs d'école (PE), par manque de formation et absence de spécialisation, ne possèdent pas les savoirs nécessaires et suffisants pour la mise en œuvre d'un enseignement consistant. Rejetant une posture prescriptive nous souhaitons comprendre les mécanismes et déterminants de l'enseignement ordinaire.

Nous l'abordons en prenant appui sur l'observation d'une modalité d'enseignement spécifique, celle d'un enseignement par «débat d'idées » (Deriaz, Poussin \& Gréhaigne 1998). L'intérêt de cette modalité est, qu'en s'appuyant sur un «geste didactique de métier » (Brière-Guenoun, 2017) comparable à celui à l'œuvre dans l'enseignement d'autres disciplines de la polyvalence (Soulé, Tozzi \& Bucheton, 2012), elle est en mesure de mettre à jour quelques-uns des savoirs professionnels des PE permettant de saisir la spécificité de cet enseignement à ce niveau de la scolarité, en dehors de tout jugement a prioriste sur ce qu'il conviendrait d'enseigner ou «en terme de manque à savoir des enseignants » (Amade-Escot, 2014). Nous développons cette étude selon une perspective didactique en nous inscrivant dans le cadre théorique de l'Action Conjointe en Didactique (Sensevy \& Mercier, 2007).

L'enseignement par "débat d'idées », parce qu'il s'appuie sur une modalité d'enseignement proche de certaines modalités en usage à l'école élémentaire est susceptible de dépasser les obstacles didactiques que font naître une approche techniciste et mécaniciste (Mérand, 1989) de l'enseignement. L'approche par l'épistémologie pratique apparaît structurante. En effet, elle analyse l'enseignement-apprentissage sur la base d'observation des actions conjointes du professeur et des élèves qui opèrent « dans le feu de l'action, c'est à dire dans des conditions qui excluent la distance, le recul, le survol, le délai, le détachement» (Bourdieu, 1980, p. 137) [et] façonnent la manière dont chacun définit la situation » (Amade-Escot, 2019). Comment les élèves construisent-ils leurs connaissances dans le feu de l'action et avec l'aide de l'enseignant? Les questions de recherche qui orientent notre travail sont les suivantes. Quelles procédures de régulation didactique émergent lors d'un enseignement des JSC par débats d'idées ? Diffèrentelles d'un enseignement ordinaire? Quels peuvent en être les motifs? Cette étude a pour but d'enrichir l'étude de 
Épistémologies pratiques du professeur et des élèves. Une étude de l’enseignement par d...

« l'épistémologie pratique du professeur et des élèves » dans un contexte de références fluctuantes non stabilisées des programmes en EPS à l'école élémentaire.

\section{Méthodologie}

L'étude est réalisée dans une classe de CM1-CM2 (élèves de 10-12 ans) avec une PE de formation littéraire et non spécialiste de l'EPS. Elle s'est inscrite dans un travail d'ingénierie didactique, construite en deux temps. Un premier temps d'observation de l'enseignement ordinaire (année 1) suivie d'un second temps, fondée sur une proposition d'ingénierie didactique (année 2). Cette dernière, fondée sur les observations préalables, a consisté en un cycle long (12 séances au lieu de 6). Elle organisait l'enseignement sur l'apprentissage de stratégies de jeu développées à l'occasion de matchs inter-équipes - équipes stables tout au long du cycle - et reproduits à chaque séance. Chaque match donnait lieu à un relevé d'observations chiffrées, réalisé par les élèves eux-mêmes. Si les thèmes d'enseignement ont été élaborés lors d'échanges entre le chercheur et l'enseignante, leur mise en œuvre effective est restée de sa responsabilité et de son autonomie.

Nous avons observé les pratiques de l'enseignante et des élèves et les contenus réellement enseignés. Les séances ont été filmées à l'aide de deux caméras, l'une en plan large sur l'ensemble du gymnase, l'autre située au milieu des élèves et enseignant. L'une d'elle est couplée à un micro-cravate porté par l'enseignante. Ce dispositif permet d'avoir plus spécifiquement accès au discours de l'enseignante. Les données recueillies ont donné lieu à une transcription du discours de l'enseignante. Les modes de réalisation des élèves sont transcrits sous forme de «configurations de jeu » situant dans l'espace, le porteur de balle ( $\mathrm{PdB})$, les non porteurs de balle (NpdB), les défenseurs. L'ensemble de ces données sont ensuite regroupées sous formes de «chroniques » (Brousseau, 1979) permettant de situer quelles observations sont à l’origine de quelles régulations.

\section{Résultats}

Nous présentons les résultats sous forme d'un tableau synthétique rapportant les données principales de compréhension des deux séquences d'enseignement, la première issue de l'observation de l'enseignement ordinaire, la seconde développée lors de l'ingénierie didactique. Puis nous exposons deux situations critiques didactiques à l’origine de deux procédures de régulation didactique significatives (tableau 1). 
Actes de la 11ème Biennale de l'ARIS : Former des citoyens physiquement éduqués. Axe 2 - Vers une éducation physique de qualité, 10.25518/sepaps20.354

Tableau 1 - Procédures de régulation didactique

\begin{tabular}{|c|c|c|}
\hline & Jeu de la passe à 10 & Balle au capitaine vers le basket-ball \\
\hline $\begin{array}{l}\text { Référence en } \\
\text { cours }\end{array}$ & Apprendre à faire des passes. & $\begin{array}{l}\text { Apprendre à faire progresser la balle collectivement pour } \\
\text { l'amener vers l'avant. }\end{array}$ \\
\hline $\begin{array}{l}\text { Théorie } \\
\text { implicite de } \\
\text { l'enseignante }\end{array}$ & $\begin{array}{l}\text { A l'école les jeux collectifs servent à apprendre } \\
\text { à faire des passes. } \\
\text { A chaque séance un nouveau jeu est présenté. }\end{array}$ & $\begin{array}{l}\text { Elle s'appuie sur sa culture sportive reposant sur une } \\
\text { connaissance certaine du football. Régulation : « au foot c'est } \\
\text { pareil quand tu as un joueur qui dribble et que tu ne peux plus } \\
\text { avancer, qu'est-ce qu'il fait? Il passe.» }\end{array}$ \\
\hline $\begin{array}{l}\text { Relevé des } \\
\text { régulations } \\
\text { didactiques }\end{array}$ & $\begin{array}{l}\text { Absence de régulation en cours d'activité, en } \\
\text { cours de jeu. L'enseignante le signale lors de } \\
\text { l'entretien post-séance : « je ne sais pas quoi } \\
\text { dire lorsque j'observe... ». } \\
\text { Une régulation en fin de jeu ; une remédiation } \\
\text { prévue à l'avance: } \\
\text { Une tâche nouvelle qui entraîne une rupture du } \\
\text { contrat didactique sur la base « d'une } \\
\text { fragmentation du savoir » (Devos-Prieur, 2006). }\end{array}$ & $\begin{array}{l}\text { Des régulations en jeu. } \\
\text { Par sa propre lecture du jeu, elle donne les solutions à appliquer } \\
\text { en s'appuyant sur la fonction perlocutoire du langage. } \\
\text { Puis des régulations ont lieu entre chaque match et chaque débat } \\
\text { d'idées. }\end{array}$ \\
\hline $\begin{array}{l}\text { Résoudre les } \\
\text { conflits du } \\
\text { Contrat } \\
\text { Didactique }\end{array}$ & $\begin{array}{l}\text { L'Incident Critique Didactique } \\
\text { Les passes sont très difficiles à réaliser. } \\
\text { Beaucoup de joueurs (partenaires et } \\
\text { adversaires sont agglutinés autour du PdB) }\end{array}$ & $\begin{array}{l}\text { Au cours d'une situation d'apprentissage de la balle au capitaine, } \\
\text { souvent le jeu s'immobilise lorsque le PdB s'arrête et les NPdB } \\
\text { avec. }\end{array}$ \\
\hline
\end{tabular}

\section{Présentation d'un Incident Critique Didactique (I.C.D.) au cours de l'enseignement ordinaire}

Les ICD ont été définis par Amade-Escot \& Marsenach (1995). L’analyse a été réalisée au cours de la 4ème séance dont le thème portait sur la passe à 10 . Au cours de la phase de verbalisation qui suit la première phase de jeu, l'enseignante, avec l'aide de l'élève arbitre, fait remarquer « un essaim d'abeilles » (jeu en grappe) entraînant un blocage du jeu. Elle invite les élèves à s'exprimer. Un échange animé s'engage entre les élèves. Cet échange montre d'un côté les joueurs, essentiellement filles, qui précisent être démarquées en jeu, mais ne reçoivent jamais la balle :

- Élève : Ben euh dans les sans maillots il y en a qui sont pas démar... enfin il y a qui sont démarqués, juste, juste devant eux pis ils vont chercher hyper loin.

- Élèves : Oh ouais!

- Maître : J'ai entendu Chloé plusieurs fois qui disais "Eh mais, moi je suis démarquée". Ça veut bien dire ce que ça veut dire.

- Élève : Ben oui.

- Maître : Ben ouais, ben ouais. Qu'est-ce que tu as constaté Imane?

- Imane : Pourtant j'ai dit à Dylan, ben voilà ... et tout ça.

- Maître : Vous veniez à peine de commencer Imane m'a dit de suite Madame, ça recommence ; ça fait un essaim d'abeilles. 
Épistémologies pratiques du professeur et des élèves. Une étude de l'enseignement par d...

De l'autre côté, un élève avance l'argument qu'être démarqué ne suffit pas, il faut aussi se déplacer pour recevoir la balle :

- Élève 3 : Peut-être se déplacer là s'il y a personne qui bouge pour aller chercher la balle euh, ben c'est pas normal non plus ...

- Élève : Ben non.

- Maître : Et alors après.

- Élève : Si on est placé.

- Élève 3 : ... lorsqu'on est placé il faut bien aller chercher la balle aussi.

L'échange fait apparaître deux positions. L'enseignante débloque la discussion avec une formulation ambiguë, surprenante : «... ce que veut dire, D. c'est qu'il faut bien faire la différence entre le moment où vous attaquez c'està-dire c'est vous qui avez la balle et le moment où vous défendez, c'est à dire le moment où il faut récupérer la balle. C'est ça D. ?». Cette régulation, prise dans «le feu de l'action » montre une impossibilité de prise en charge de la contradiction faute d'une identification de l'obstacle de jeu. L'enseignante a en tête à ce moment-là, l'avancée de la séance et la proposition de remédiation déjà prévue à l'avance dans le plan de séance. « Alors, puisque effectivement le fait d'attraper la balle, ça pose problème, on va faire un petit exercice là-dessus ». Elle met alors en place un exercice de lancer de balle, où les élèves espacés de 4 à 5 mètres, doivent se lancer et rattraper la balle.

\section{Présentation d'un I.C.D. au cours de l'enseignement par débat d'idées}

Celui-ci a lieu lors de la $5^{\text {ème }}$ séance. Le thème de la séance: la balle au capitaine. Chacune des quatre équipes rencontre l'autre, au cours de trois matchs suivis d'un débat d'idées, durant lesquels les élèves doivent réajuster leur stratégie de jeu.

Alors qu'une des équipes a formulé la stratégie suivante : « Là, on va mettre un capitaine. Ça sera Corentin. Déjà on va mettre Saphia pour pas (inaudible) ... Là, ce sera moi et Pauline, pour empêcher les autres. Bon on va dribbler pour passer la balle à Saphia. Et Perrine elle va, elle va empêcher l'autre capitaine d'avoir la balle (équipe Jaune). En cours de jeu, lors des phases de remise en jeu, les NPdB se placent loin devant, les PdB éprouvent beaucoup de difficultés à transmettre la balle. L'enseignante intervient alors fréquemment : « Non!! Eh, les filles! Vous voyez rien de ce qui se passe ! Bougez! ... Suivez l'action, les arbitres! (Jeu)... Les filles, vous savez courir ! Suivez l'action! Allez Alex! (Jeu). Naomi, bravo pour la réception, hein! Bravo pour la réception, c'est bien! (Jeu). Allez, vite, vite, vite! Placez-vous! Dommage ! Ouais, bien ça ! ... dommage ! (Jeu). Eh! Il est là, tout seul ! Comment voulez-vous qu'il jette la balle là-bas ! Les jaunes, réagissez !»

\section{Discussion}

L'analyse de ces deux I.C.D. permet de mettre en évidence des Procédures de Régulation Didactique (P.R.D.) de nature tout à fait différente. Au cours de l'enseignement ordinaire, reposant sur un modèle d'enseignement de modèles gestuels (Amade-Escot, 1988), ici l'échange de la balle dans sa décomposition entre le lancer et le réceptionner, les P. R. D. se déroulent au cours de moments de verbalisation ritualisés. A la suite de quoi l'enseignante met en place un exercice d'apprentissage décroché, et souvent décontextualisé (Devos-Prieur, 2006). Ces PRD reposent sur une analyse faite par l'enseignante en termes de prérequis non maîtrisés par les élèves (puisque le fait d'attraper la balle ça pose problème ...). L'enseignement reposant sur la modalité du débat d'idées enclenche un enseignement par dévolution d'un problème, une progression rapide de la balle vers la cible, en présence d'adversaire. Les PRD émergent alors pendant le déroulement même des phases de jeu et s'appuient sur la valence perlocutoire du langage (Austin, 1962). Ces PRD visent la construction d'une règle d'action, le déplacement des NPdB, même lorsque la balle est à l'arrêt, tout en tenant compte du contexte fluctuant des rapports d'opposition entre les partenaires et les adversaires, aller vers 
Actes de la 11ème Biennale de l'ARIS : Former des citoyens physiquement éduqués. Axe 2 - Vers une éducation physique de qualité, 10.25518/sepaps20.354

l'avant et se démarquer/revenir soutenir le PdB.

Nous attribuons cette transformation significative de l'activité enseignante à la dimension "geste didactique de métier» (Brière-Guenoun 2017) qu'est l'enseignement pas débats d'idées. En s'appuyant sur ce geste, acquis et maîtrisé par cette enseignante expérimentée au cours des autres enseignements disciplinaires de la polyvalence, elle a permis de révéler, de donner toute sa dimension en convoquant alors ses connaissances personnelles de la pratique de référence. Cette modalité a libéré l'enseignante d'un carcan professionnel. Elle s'autorise spontanément à intervenir verbalement pendant le cours du jeu pour réguler l'activité des élèves. L'étude souligne également combien l'appui sur un geste didactique de métier permet de surmonter quelques-uns des obstacles de l'enseignement ordinaire et permet à l'enseignante d'engager ses élèves dans le processus d'apprentissage.

L'abord par l'épistémologie pratique met ici à jour les arrière-plans, culturel et institutionnel qui pèsent sur le processus didactique (Amade-Escot, 2019). Il permet également de comprendre l'effet de levier qu'a entraîné une procédure d'enseignement innovante.

\section{Conclusion}

L'objet de notre étude vise à explorer quelques-unes des dimensions de l'épistémologie pratique du professeur et des élèves pour l'analyse du processus d'enseignement-apprentissage en EPS à l'école élémentaire. Cette étude met à jour l'effet de levier qu'entraîne l'introduction d'un geste didactique de métier familier chez les PE : le débat d'idées. Cette modalité d'enseignement, importée et maîtrisée dans d'autres disciplines scolaires par l'enseignante facilite son appropriation de cette procédure d'enseignement innovante.

L'étude de l'épistémologie pratique, par une approche systémique du processus d'enseignement-apprentissage facilite une perception globale des différentes composantes du métier et de leurs interactions permanentes avec l'activité des élèves. Elle souligne une dimension spécifique du métier de professeur d'école. A une maîtrise moins experte des savoirs à enseigner répond une autre expertise réelle des savoirs comment enseigner, issue de la pratique polyvalente des disciplines. Cette étude ouvre la voie vers une meilleure compréhension de la dynamique curriculaire en EPS à l'école, de la maternelle au baccalauréat.

\section{Bibliographie}

Amade-Escot, C. (1988). Stratégies d'enseignement en EPS : contenus proposés, conceptions de l'apprentissage et perspectives de différenciation. In P. Chifflet (Ed.), Méthodologie et didactique de l'éducation physique et sportive (pp.131-142). Grenoble, édition AFRAPS.

Amade-Escot, C. (2014). De la nécessité d'une observation didactique pour accéder à l'épistémologie pratique des professeurs. Revue de recherches en éducation, 19, 18-29.

Amade-Escot, C. (2019). Épistémologies pratiques et action didactique conjointe du professeur et des élèves. Éducation \& Didactique, 13(1), 109-114.

Amade-Escot, C., \& Marsenach, J. (1995). Didactique de l'éducation physique et sportive. Grenoble : La Pensée Sauvage.

Austin, J.-L. (1962). Quand dire c'est faire. Paris : Seuil.

Brière-Guenoun, F. (2017). Instruire les gestes didactiques de métier. Rennes : Presses Universitaires de Rennes.

Brousseau, G. (1979). L’observation des activités didactiques. Revue française de pédagogie, 45, 130-139. 
Épistémologies pratiques du professeur et des élèves. Une étude de l’enseignement par d...

Cicurel, F. (2019). Pour une archéologie des pratiques enseignantes. Éducation \& Didactique, 13(1), 116-121.

Deriaz, D., Poussin, B., \& Gréhaigne, J. F. (1998). Le débat d'idées. Éducation physique et Sport, 273, 80-82.

Devos-Prieur, O. (2006). Rapports aux savoirs des professeurs d'école et développement des contenus en éducation physique. Étude comparée de quatre cycles de basket-ball au cours moyen. Thèse de doctorat de sciences de l'éducation, non publiée. Toulouse : Université Paul Sabatier.

Mérand, R. (1989). La rénovation des contenus d'enseignement : jeux sportifs collectifs au collège. Revue Française de Pédagogie, 89, 11-14.

Sensevy, G., \& Mercier, A. (Eds.) (2007). Agir ensemble. L'action didactique conjointe du professeur et des élèves. Rennes : Presses Universitaires de Rennes.

Soulé, Y., Tozzi, M., \& Bucheton, D. (2012). La littérature en débat. Montpellier : Scéren.

PDF automatiquement généré le 2023-04-26 15:49:32

Url de l'article : https://popups.uliege.be/sepaps20/index.php?id=354

Publié par ULiège Library en Open Access et distribué suivant les termes et les conditions de la licence CC-BY (https://creativecommons.org/licenses/by/4.0/deed.fr) 